\section{Impacto do incentivo ao aleitamento materno entre mulheres trabalhadoras formais}

\author{
The impact of breastfeeding promotion in women \\ with formal employment
}

\begin{abstract}
This study focused on programs to promote breastfeeding in order to prevent early weaning of working mothers' infant children. A non-randomized intervention study was conducted using a survey of mothers who had returned to work after childbirth, including both participants and non-participants in a program to promote breastfeeding. The sample consisted of 200 mothers of infants ranging from 6 to 10 months of age. Factors associated with early weaning were analyzed with the chi-square and Fisher's exact tests and multiple logistic regression ( $\alpha=0.05)$. The results showed statistical differences between the groups in relation to exclusive breastfeeding $(p<0.0001)$ and breastfeeding $(p<0.0001)$. There was a statistically significant difference ( $p=0.0056$ ) between the groups in relation to time between childbirth and return to work. There was no difference between the end of maternity leave and weaning time. Mothers that were unable to nurse their infants during the work shift showed 4.98 times higher odds (95\%CI: 1.27-19.61) of weaning them before the fourth month of age.
\end{abstract}

Breast Feeding; Weaning; Parental Leave; Women Working; Social Support

\author{
Aline Alves Brasileiro ${ }^{1}$ \\ Rosana de Fátima Possobon 1 \\ Karina Camilo Carrascoza 1 \\ Gláucia Maria Bovi Ambrosano 1 \\ Antônio Bento Alves de Moraes ${ }^{1}$
}

\section{Introdução}

Apesar do crescente incentivo ao aleitamento materno, iniciado na década de 1980, o Brasil ainda registra baixos índices de amamentação exclusiva ao sexto mês de vida da criança. Uma recente pesquisa do Ministério da Saúde aponta para um importante aumento nos índices do aleitamento materno exclusivo ao quarto mês de vida, de $35,5 \%$ no ano de 1999 para $51,2 \%$ em 2008. Entretanto, esse índice, ao sexto mês, praticamente não foi alterado 1 .

O incentivo ao aleitamento materno tem sido o objetivo de diversos grupos de profissionais da saúde, em diferentes localidades do Brasil. Esses grupos relatam índices satisfatórios de adesão das mães a essa prática, mostrando que entre $35 \%$ e $38,6 \%$ das crianças são mantidas em aleitamento materno exclusivo até o sexto mês de idade 2,3,4. Índice superior foi relatado por Carrascoza 5 , que encontrou $47,5 \%$ das crianças participantes de um grupo de incentivo ao aleitamento materno amamentadas de forma exclusiva até o sexto mês de idade. Segundo o autor, esse índice se deve às atuações sistemáticas de uma equipe interdisciplinar, que acompanha a mãe e o lactente durante os primeiros seis meses após o parto, por meio de nove encontros, além de sessões individuais para esclarecimento de dúvidas, treino da prática da amamentação e tratamento de problemas de mama. 
Ainda que esses índices 2,3,4,5 estejam abaixo do preconizado pela Organização Mundial da Saúde (OMS), que pretendia alcançar $100 \%$ de crianças amamentadas de forma exclusiva ao sexto mês, ainda no ano de 2001 6, eles mostramse elevados quando comparados com dados de estudos investigativos sobre o índice de aleitamento entre crianças brasileiras cujas mães não estão vinculadas a programas de incentivo e que, portanto, não recebem auxílio formal para a manutenção desta prática 6,7. Dados do Ministério da Saúde em $2009{ }^{1}$ mostram que, em média, 9,3\% das mulheres brasileiras amamentam seus filhos de forma exclusiva até o sexto mês de vida.

Assim, pode-se dizer que as estratégias de promoção e incentivo ao aleitamento materno têm sido importantes para a manutenção da amamentação exclusiva e conseqüente prevenção do desmame precoce. Dentre essas estratégias, é importante citar a "Iniciativa Hospital Amigo da Criança”, criada em 1990, pelo Fundo das Nações Unidas para a Infância (UNICEF) e OMS. O principal objetivo dessa iniciativa é mobilizar os funcionários de hospitais e maternidades a seguir condutas e rotinas adequadas à prática do aleitamento. Para obter o título de "Hospital Amigo da Criança”, a instituição deve cumprir dez passos de incentivo ao aleitamento, sendo que o décimo é encorajar a formação de grupos de apoio à amamentação, para onde as mães devem ser encaminhadas logo após a alta do hospital ou ambulatório 8 .

Dentre os fatores de risco ao aleitamento materno podem ser destacados o uso de chupeta ${ }^{9}$, a primiparidade 10,11,12 e o trabalho materno 2,13,14. Pesquisas mostram que a crescente participação da mulher no mercado de trabalho tem elevado a ocorrência da prática do desmame precoce 15,16. Os resultados desses estudos sugerem que o incentivo ao aleitamento materno poderia minimizar os riscos de desmame precoce entre mães trabalhadoras.

O objetivo deste estudo foi investigar se mães trabalhadoras formais, participantes de um programa de incentivo ao aleitamento materno, mantêm a amamentação por mais tempo do que mães que não têm este apoio após o retorno ao trabalho.

\section{Metodologia}

A amostra foi constituída por 200 díades mãe/ lactente, sendo 100 participantes (Grupo A) e 100 não-participantes (Grupo B) de um programa de incentivo ao aleitamento materno, desenvolvido na cidade de Piracicaba, São Paulo. O cálculo da amostra foi realizado segundo Demidenko 17,18, considerando-se o poder do teste de 0,80 , nível de significância de 0,05 e odds ratio de 3 .

Em ambos os grupos foram incluídas somente as mães trabalhadoras formais, ou seja, que possuíam vínculo empregatício ("carteira assinada”) e que retornaram ao trabalho antes do filho completar seis meses de vida. Os dados foram coletados quando as crianças tinham entre $6 \mathrm{e}$ 10 meses de idade. Foram excluídas as mães que desempenhavam funções não regulamentadas pela Consolidação das Leis Trabalhistas (CLT) 19, por não serem contempladas com os benefícios de incentivo ao aleitamento materno concedidos por esta regulamentação. Além disso, também foram excluídas as díades que apresentaram algum problema de saúde nos períodos pré e pósparto imediato, bem como as mães que tiveram filhos gêmeos, prematuros ( $<37$ semanas) ou com baixo peso ao nascer $(<2.500 \mathrm{~g})$. As mães que compuseram o Grupo B não podiam ter participado de qualquer programa de incentivo ao aleitamento materno em nenhum momento da gravidez ou puerpério.

Para a composição das amostras, as mães do Grupo A foram selecionadas aleatoriamente durante a sua participação no programa de incentivo ao aleitamento. A adesão das mães ao programa acontece de forma voluntária e o acesso é livre para a população em geral. Esse programa é oferecido por uma universidade de odontologia, onde a população pode ser atendida de forma gratuita. A divulgação do programa é feita nos postos de saúde e unidades de saúde da família, em consultórios de ginecologistas e, em algumas ocasiões, por meio das mídias jornal e rádio. Muitas mães são encaminhadas pelos profissionais de saúde que as atendem. As mães interessadas em participar fazem inscrição pessoalmente ou por telefone e ingressam no programa ainda no período gestacional. Ao longo dos seis meses de programa, verifica-se uma perda de cerca de $12,5 \%$ das mães.

Para compor o Grupo B, as mães foram abordadas durante uma campanha de vacinação infantil, realizada na cidade de Piracicaba, no dia 10 de junho de 2006. Considerando que as crianças não estão distribuídas uniformemente nas várias unidades de vacinação (conglomerados), adotou-se o sorteio em dois estágios, com probabilidade proporcional ao tamanho dos conglomerados. No primeiro estágio foram sorteadas as unidades de vacinação e, no segundo, as crianças em cada unidade. Sorteou-se três unidades e o número de crianças cujas mães seriam abordadas por unidade foi definido considerando o total de 40 unidades de vacinação do ano anterior e a estimativa de vacinar 24 mil crianças de 0-5 anos. Nessas três unidades sorteadas, a expecta- 
tiva era vacinar 1.200 crianças com idade inferior a 1 ano.

No programa de incentivo ao aleitamento, a díade mãe-lactente era acompanhada durante os seis primeiros meses de vida, participando de nove encontros, conduzidos por uma equipe interdisciplinar composta por cirurgião-dentista, fonoaudiólogo, nutricionista e psicólogo. Nesses encontros e em sessões individuais, quando necessário, a equipe disponibilizava apoio informativo, instrumental e afetivo para que a mãe adquirisse condições para a manutenção da amamentação natural.

Para coleta das informações utilizou-se um roteiro estruturado de entrevista que permitiu identificar fatores tais como nível socioeconômico, idade da mãe e do pai, estado civil, tipo de parto, variáveis relacionadas aos períodos peri e pós-natal, época de retorno da mãe ao trabalho, condições do trabalho materno e época de desmame.

Para efeito deste estudo, os seguintes conceitos foram utilizados, segundo a definição da OMS 20:

- Aleitamento materno exclusivo (AME): a criança recebe leite materno diretamente de sua mãe ou leite materno ordenhado. Nenhum outro líquido ou sólido é oferecido à criança, com exceção de gotas ou xaropes de vitaminas, suplementos minerais ou medicamentos;

- Aleitamento materno (AM): a criança recebe leite materno direto da mama ou ordenhado, independentemente da presença de outro alimento na dieta da criança;

- Desmame (D): a criança não mais recebe o leite materno.

Os resultados foram analisados por meio de análise descritiva, dos testes estatísticos de quiquadrado, exato de Fisher e análise de regressão logística múltipla pelo procedimento stepwise forward. Foram testadas no modelo as variáveis com $\mathrm{p}<0,15$ na análise bivariada, permanecendo as que se ajustaram ao modelo com $\mathrm{p} \leq 0,05$. Todas as análises foram realizadas no programa estatístico SAS 2008, versão 9.1 (SAS Inst., Cary, Estados Unidos).

Este estudo foi realizado de acordo com as Normas e Diretrizes Éticas da Resolução $n^{o}$. 196/1996 do Conselho Nacional de Saúde do Ministério da Saúde, e aprovado pelo Comitê de Ética em Pesquisa da Faculdade de Odontologia de Piracicaba, Universidade Estadual de Campinas (UNICAMP, protocolo $\mathrm{n}^{\circ}$. 073/2004). Todas as mães participantes assinaram uma cópia do Termo de Consentimento Livre e Esclarecido para Pesquisa.

\section{Resultados}

Na Tabela 1 são apresentadas as principais características da população estudada, mostrando informações sobre os aspectos pessoais e demográficos, o estado civil, os períodos peri e pósnatal e as características do trabalho materno. É importante ressaltar que os dois grupos apresentaram características semelhantes, diferindo estatisticamente em relação aos seguintes itens: escolaridade da mãe, idade do pai, primiparidade, tipo de parto, tempo de início da amamentação e permanência em alojamento conjunto.

Ao se comparar a época de retorno da mãe ao trabalho entre os dois grupos, foi observada diferença significativa $(p=0,0056)$. Entre as mães do grupo não-participantes de um programa de incentivo ao aleitamento materno (Grupo B), o retorno ao trabalho ocorreu, predominantemente, no terceiro (21\%), no quarto (35\%) e no quinto (27\%) meses de vida do lactente. As mães do grupo participantes desse programa (Grupo A) retornaram ao trabalho, principalmente, no quarto (43\%) e no quinto (42\%) meses.

Os índices de aleitamento materno e aleitamento materno exclusivo, durante os primeiros seis meses de vida, foram significativamente superiores entre as crianças cujas mães participaram do programa ( $\mathrm{p}<0,001)$, em relação a todos os meses avaliados. Esses índices ficaram em torno de $90 \%$ e $65 \%$ de aleitamento materno exclusivo no primeiro mês, nos grupos A e B, respectivamente, passando a $33 \%$ e $6 \%$ no sexto mês. Se analisados os índices de aleitamento materno, eles foram de $97 \%$ e $88 \%$ no primeiro mês para $77 \%$ e $45 \%$ no sexto nos grupos A e B, respectivamente.

Pela análise bivariada verificou-se que a manutenção do aleitamento materno exclusivo além do quarto mês de vida sofreu influência dos seguintes fatores: participação no programa de incentivo ao aleitamento, mães com idade superior a 28 anos e ocupando cargos de nível superior, oferecimento de leite materno ordenhado em copo, não oferecimento de fórmula infantil na mamadeira e conseguir amamentar durante a jornada de trabalho (Tabela 2).

A Tabela 3 apresenta os resultados da análise de regressão logística múltipla das variáveis relacionadas ao aleitamento materno exclusivo no quarto mês. Pode-se observar que as mães do grupo que não participaram do programa de incentivo têm 8,84 vezes mais chances de desmamar antes do quarto mês se comparadas com as mães do outro grupo. As mães que não conseguem amamentar seus filhos durante a jornada de trabalho têm 4,98 vezes mais chances de desmamá-los antes do quarto mês. As crianças 
Análise univariada das características da população estudada.

\begin{tabular}{|c|c|c|c|}
\hline & $\begin{array}{l}\text { Participantes } \\
\text { de um programa } \\
\text { de incentivo } \\
\%(n=100)\end{array}$ & $\begin{array}{l}\text { Não participantes } \\
\text { de um programa } \\
\text { de incentivo } \\
\%(n=100)\end{array}$ & Valor de $p$ \\
\hline \multicolumn{4}{|l|}{ Pessoais e demográficas } \\
\hline Nível socioeconômico alto & 56 & 53 & 0,0831 \\
\hline Pai com mais do que o 1o grau completo & 74 & 76 & 0,1137 \\
\hline Mãe com mais do que o 1ㅇ grau completo & 93 & 77 & 0,0015 \\
\hline Pai com idade inferior a 25 anos & 11 & 25 & 0,0099 \\
\hline Mãe com idade inferior a 25 anos & 24 & 34 & 0,1191 \\
\hline Primíparas & 76 & 63 & 0,0458 \\
\hline Estado civil antes do parto & & & 0,0299 \\
\hline Casada/União estável & 98 & 91 & \\
\hline Solteira/Separada/Divorciada & 2 & 9 & \\
\hline \multicolumn{4}{|l|}{ Período perinatal e pós-natal } \\
\hline Parto normal & 16 & 34 & 0,0032 \\
\hline Início da amamentação até 4 horas após o parto & 39 & 55 & 0,0234 \\
\hline Permanência em alojamento conjunto & 88 & 74 & 0,0116 \\
\hline \multicolumn{4}{|l|}{ Características do trabalho } \\
\hline 30 minutos por turno & 51 & 38 & 0,0643 \\
\hline Creche interna na empresa & 4 & 9 & 0,1515 \\
\hline Creche externa paga pela empresa & 7 & 5 & 0,5515 \\
\hline Posto de coleta de leite materno & 10 & 4 & 0,0963 \\
\hline
\end{tabular}

que recebem fórmula infantil na mamadeira têm 46,5 vezes mais chances de ser desmamadas antes do quarto mês.

Não foi encontrada relação estatística entre a época de retorno ao trabalho e o índice de aleitamento materno exclusivo aos seis meses de vida da criança nos dois grupos. Assim, a manutenção da prática do aleitamento materno exclusivo independe do período de retorno da mãe ao trabalho.

A ocorrência de desmame entre as mães do Grupo B foi mais freqüente no primeiro $(21,4 \%)$ e no quarto $(21,4 \%)$ meses de vida da criança. Entre as mães do Grupo A, a interrupção do aleitamento materno foi mais prevalente no quarto $(21,7 \%)$, no quinto $(26,8 \%)$ e no sexto $(21,7 \%)$ meses após o parto. A análise estatística permitiu observar maior precocidade do desmame entre as mães do grupo que não participou do programa de incentivo ao aleitamento materno $(\mathrm{p}=0,0009)$.

\section{Discussão}

Dentre as variáveis de caracterização da amostra, três poderiam indicar maiores chances de sucesso na amamentação entre as mães que receberam apoio para a prática do aleitamento: o grau de escolaridade superior ao primeiro grau completo, a presença do companheiro (mães casadas ou em união estável) e a permanência em alojamento conjunto. A literatura aponta para uma relação positiva entre mães com alto grau de escolaridade e a manutenção da amamentação 10,21,22,23,24, justificada pelo maior acesso à informação sobre os benefícios desta prática. Entretanto, pesquisas realizadas pelo Ministério da Saúde afirmam que esse não é um fator de proteção ao aleitamento, uma vez que não foi encontrada diferença estatística entre o índice de aleitamento e os diversos graus de escolaridade apresentados pela população estudada 1 .

Diversos autores mostram a importância da presença do companheiro como um fator de apoio à instalação e manutenção da prática do aleitamento 13,25 . Silveira \& Lamounier 26 relatam que a chance de uma criança desmamar precocemente é 1,46 vez maior na ausência do pai. No 
Análise bivariada da manutenção de aleitamento materno exclusivo em relação às características do trabalho materno e alimentação da criança.

\begin{tabular}{|c|c|c|c|c|c|c|c|}
\hline \multirow[t]{3}{*}{ Características } & \multirow[t]{3}{*}{ n } & \multirow[t]{3}{*}{$\%$} & \multicolumn{4}{|c|}{ Aleitamento materno exclusivo } & \multirow[t]{3}{*}{ Valor de $p$} \\
\hline & & & \multicolumn{2}{|c|}{ Até o 4 o mês } & \multicolumn{2}{|c|}{ Após o 4o mês } & \\
\hline & & & $\mathbf{n}$ & $\%$ & $\mathbf{n}$ & $\%$ & \\
\hline Grupo & & & & & & & $<0,0003$ \\
\hline Participantes de um programa de incentivo & 100 & 50,00 & 63 & 63,00 & 37 & 37,00 & \\
\hline Não-participantes de um programa de incentivo & 100 & 50,00 & 93 & 93,00 & 7 & 7,00 & \\
\hline Idade da mãe (anos) & & & & & & & 0,0041 \\
\hline Até 28 & 87 & 43,90 & 76 & 87,40 & 11 & 12,60 & \\
\hline$>28$ & 111 & 56,10 & 78 & 70,30 & 33 & 29,70 & \\
\hline Estado civil antes do parto & & & & & & & 0,4634 \\
\hline Casada/União estável & 188 & 94,95 & 145 & 77,10 & 43 & 22,90 & \\
\hline Solteira/Separada/Divorciada & 10 & 5,05 & 9 & 90,00 & 1 & 10,00 & \\
\hline Nível do cargo ocupado & & & & & & & 0,0387 \\
\hline Superior & 82 & 41,40 & 58 & 70,70 & 24 & 29,30 & \\
\hline Nível médio & 87 & 43,95 & 69 & 79,30 & 18 & 20,70 & \\
\hline Nível intermediário & 29 & 14,65 & 27 & 93,10 & 2 & 6,90 & \\
\hline Voltou ao trabalho & & & & & & & 0,1310 \\
\hline Do 1o mês ao 2o mês & 21 & 10,60 & 17 & 80,95 & 4 & 19,05 & \\
\hline Do 3o mês ao 4o mês & 108 & 54,55 & 89 & 82,40 & 19 & 17,60 & \\
\hline Ao 5o mês & 69 & 34,85 & 48 & 69,60 & 21 & 30,40 & \\
\hline Recebeu amamentação durante a jornada de trabalho & & & & & & & $<0,0001$ \\
\hline $\operatorname{Sim}$ & 46 & 23,20 & 25 & 54,35 & 21 & 45,65 & \\
\hline Não & 152 & 76,80 & 129 & 84,90 & 23 & 15,10 & \\
\hline Recebeu leite materno no copo & & & & & & & $<0,0001$ \\
\hline Sim & 33 & 16,70 & 13 & 39,40 & 20 & 60,60 & \\
\hline Não & 165 & 83,30 & 141 & 85,45 & 24 & 14,55 & \\
\hline Recebeu fórmula infantil na mamadeira & & & & & & & 0,0000 \\
\hline Sim & 108 & 54,55 & 107 & 99,10 & 1 & 0,50 & \\
\hline Não & 90 & 45,45 & 47 & 52,20 & 43 & 47,80 & \\
\hline
\end{tabular}

entanto, algumas atitudes paternas tais como o ciúme decorrente da maior proximidade física e emocional entre mãe e criança e o fato do pai não participar ativamente da alimentação do filho, podem criar conflitos que desencorajam a mulher quanto ao processo de lactação.

A permanência em alojamento conjunto é considerada por vários autores como um fator facilitador para o estabelecimento da prática da amamentação, uma vez que a criança pode ser levada ao peito da mãe sempre que se fizer necessário, sem que tenha de seguir a rotina de horários estabelecida pela maternidade 3,14,27,28 . Entretanto, Carrascoza ${ }^{5}$ encontrou diferença significativa ao comparar a permanência em alojamento conjunto entre dois grupos de crianças: desmamadas precocemente (antes do sexto mês de vida) e mantidas em aleitamento além dos 12 meses. Neste estudo, o desmame precoce esteve presente em $90 \%$ das mães que permanece- ram em alojamento conjunto e apenas $40 \%$ das mães que mantiveram aleitamento por mais de um ano usufruíram deste tipo de alojamento. $\mathrm{Na}$ cidade de Piracicaba, onde o presente estudo foi conduzido, há uma forte tendência em manter o lactente em alojamento conjunto, sendo que as três maternidades da cidade adotam esta prática como rotina.

Entre as mães que não receberam apoio formal para a amamentação, também foram encontradas três variáveis com potencial de influenciar positivamente a manutenção desta prática: multiparidade, parto normal e início da amamentação nas primeiras quatro horas após o parto. A literatura mostra que as primíparas têm maior chance de iniciar a amamentação, porém tendem a mantê-la por menos tempo quando comparadas às multíparas 12,25,29. Entretanto, há que se considerar que o nascimento de cada filho pode ocorrer num contexto diferente (idade da 
Tabela 3

Fatores associados ao aleitamento materno exclusivo aos quatro meses de idade, segundo regressão logística.

\begin{tabular}{|c|c|c|c|c|c|}
\hline \multirow[t]{2}{*}{ Características } & \multicolumn{2}{|c|}{$\begin{array}{l}\text { Amamentou exclusivamente } \\
\text { até o } 4 \text { o mês }\end{array}$} & \multirow[t]{2}{*}{ OR } & \multirow[t]{2}{*}{ IC95\% } & \multirow[t]{2}{*}{ Valor de $\mathrm{p}$} \\
\hline & $\mathbf{n}$ & $\%$ & & & \\
\hline \multicolumn{6}{|l|}{ Recebe fórmula infantil na mamadeira } \\
\hline Sim & 107 & 99,1 & 46,50 & $5,56-388,80$ & $<0,0001$ \\
\hline Não & 47 & 52,2 & 1,00 & & \\
\hline \multicolumn{6}{|l|}{ Grupo } \\
\hline Participantes de um programa de incentivo & 61 & 62,2 & 1,00 & & 0,0005 \\
\hline Não-participantes de um programa de incentivo & 93 & 93,0 & 8,84 & $2,11-37,11$ & \\
\hline \multicolumn{6}{|l|}{ Recebe amamentação durante a jornada de trabalho } \\
\hline Sim & 25 & 54,35 & 1,00 & & 0,0024 \\
\hline Não & 129 & 84,9 & 4,98 & $1,27-19,61$ & \\
\hline
\end{tabular}

IC95\%: intervalo de 95\% confiança; OR: odds ratio.

mãe, situação conjugal, condição socioeconômica etc.) e, portanto, apenas a experiência prévia em amamentação pode não ser um fator de proteção a esta prática 11,26.

O parto vaginal é considerado um facilitador para aleitamento materno, uma vez que permite o contato precoce entre mãe/lactente, ocorrendo a primeira mamada, na maioria dos casos, dentro da sala de parto. Por não haver o fator dor incisional e/ou o efeito pós-anestésico, relacionados com o parto do tipo cesárea, que podem se estender por várias horas após o ato cirúrgico e retardar o contato mãe/lactente, as primeiras mamadas ocorrem mais precocemente entre estas mulheres 25,30,31.

Assim sendo, ambos os grupos apresentam três variáveis consideradas como fatores de proteção à amamentação. No entanto, pode-se inferir que a mãe que participa de um programa de incentivo ao aleitamento estaria mais predisposta a amamentar. Porém, deve-se ressaltar que o presente estudo teve por objetivo investigar se a mulher trabalhadora formal continua a manter essa predisposição após o retorno ao trabalho. A maioria das mães de ambos os grupos retornou ao trabalho entre o terceiro e o quinto meses após o parto. Esse resultado já era esperado, tendo em vista a legislação trabalhista vigente no país no momento da coleta dos dados, que dava o direito a quatro meses de licença-maternidade remunerada às mulheres trabalhadoras formais. Segundo dados do Ministério da Saúde 1 , houve predomínio do aleitamento materno e aleitamento materno exclusivo entre as mulheres que usufruíam do beneficio da licençamaternidade.
A comparação da época de retorno ao trabalho entre os dois grupos mostra que as mães que receberam incentivo ao aleitamento ficaram por mais tempo afastadas do ambiente de trabalho, o que permitiu uma maior permanência com a criança. Entretanto, os resultados deste estudo sugerem não haver relação entre a época de retorno ao trabalho e a interrupção do aleitamento materno exclusivo, corroborando o estudo de Ramos et al. 9. Além disso, não houve diferença significativa entre os dois grupos em relação aos benefícios trabalhistas recebidos, referentes a dois intervalos de 30 minutos para mamada em cada período de 4 horas de trabalho, acesso à creche e posto de coleta de leite materno no local de trabalho. Dessa forma, parece importante que, além da extensão do período de licença-maternidade de quatro para seis meses, também seja disponibilizado apoio para que a mãe mantenha essa prática.

Os índices de aleitamento materno, de ambos os grupos, apresentados nos primeiros seis meses, são superiores aos resultados de um estudo realizado na cidade de São Paulo, com mulheres trabalhadoras 32 . Nesse estudo, $38 \%$ das crianças com 15 dias de vida recebiam aleitamento materno exclusivo, sendo que esta porcentagem caiu para $17 \%$ no segundo mês. No sexto mês de vida, $40 \%$ das crianças ainda recebiam leite materno, porém nenhuma delas de forma exclusiva. Damião 33 encontrou uma taxa de 11,2\% de mães trabalhadoras que mantiveram o aleitamento materno, não de forma exclusiva, até o quarto mês de vida. Índice inferior foi encontrado por Vianna et al. 34 , que relataram que apenas 5,3\% das mulheres trabalhadoras amamentavam nos 
primeiros quatro meses de vida da criança. Esses índices são semelhantes aos encontrados no presente estudo entre as mães que não participaram do programa de incentivo. Essas mulheres desmamaram mais precocemente seus filhos quando comparadas às mães que receberam informação formal sobre aleitamento.

Borges \& Philippi 35 acreditam que a insegurança materna, gerada pela aproximação da época de retorno ao trabalho, pode ser um fator de risco para a manutenção do aleitamento. A participação em programa de incentivo, no qual são disponibilizadas informações teóricas e práticas sobre a amamentação, além de suporte emocional à nutriz, pode contribuir para o aumento dos índices de aleitamento materno e aleitamento materno exclusivo entre mulheres trabalhadoras.

\section{Resumo}

Investigar se programas de incentivo ao aleitamento materno ajudam a prevenir o desmame precoce entre filhos de mães trabalhadoras. Foi realizado um estudo de intervenção não randomizado, por meio de inquérito entre mães que voltaram a trabalhar após o parto, participantes e não-participantes de um programa de incentivo ao aleitamento. A amostra consistiu de 200 mães de filhos com idades entre 6 e 10 meses. Para avaliar fatores associados ao desmame precoce, os resultados foram analisados por meio dos testes estatísticos de qui-quadrado, exato de Fisher e análise de regressão logística múltipla $(\alpha=0,05)$. Os resultados mostraram que houve diferença estatística nas taxas de aleitamento materno exclusivo $(p<0,0001)$ e de aleitamento materno ( $p<0,0001)$ entre os grupos. Apresentou diferença estatística $(p=0,0056)$ em relação à época de retorno ao trabalho entre os grupos. Não houve diferença entre o fim da licença-maternidade e a época de desmame. As mães que não conseguem amamentar seus filhos durante a jornada de trabalho têm 4,98 (IC95\%: 1,27-19,61) vezes mais chances de desmamálos antes do quarto mês.

Aleitamento Materno; Desmame; Licença Parental; Trabalho Feminino; Apoio Social

\section{Conclusão}

Os dados deste estudo apontam para uma relação favorável entre mães trabalhadoras que participam de programas de incentivo ao aleitamento e a manutenção desta prática, sugerindo a importância do apoio de profissionais de saúde treinados para a manutenção do aleitamento materno.

\section{Colaboradores}

A. A. Brasileiro colaborou na metodologia, coleta de dados, redação e revisão final do artigo. R. F. Possobon contribuiu na metodologia, redação e revisão final do artigo. K. C. Carrascoza participou na metodologia e redação. G. M. B. Ambrosano colaborou na análise estatística e revisão final. A. B. A. Moraes contribuiu na metodologia e revisão final.

\section{Agradecimentos}

Agradeço à Coordenação de Aperfeiçoamento de Pessoal de Nível Superior (CAPES) por proporcionar a realização deste trabalho. 


\section{Referências}

1. Secretaria de Atenção à Saúde, Ministério da Saúde. II Pesquisa de prevalência de aleitamento materno nas capitais brasileiras e Distrito Federal. Brasília: Ministério da Saúde; 2009.

2. Oliveira MIC, Camacho LAB, Tedstone AE. A method for the evaluation of primary health care units' practice in the promotion, protection, and support of breastfeeding: results from the State of Rio de Janeiro, Brazil. J Hum Lact 2003; 19:365.

3. Narchi NZ, Fernandes RAQ, Gomes MMF. Análise da efetividade de um programa de incentivo ao aleitamento materno exclusivo em comunidade carente na Cidade de São Paulo. Rev Bras Saúde Matern Infant 2005; 5:87-92.

4. Faleiros JJ, Kalil G, Casarin DP, Laque Jr. PA, Santos IS. Avaliação do impacto de um programa de puericultura na promoção da amamentação exclusiva. Cad Saúde Pública 2005; 21:482-9.

5. Carrascoza KC. Avaliação dos aspectos biopsicossociais que interferem na amamentação [Dissertação de Mestrado]. Campinas: Universidade Estadual de Campinas; 2007.

6. Kummer SC, Giugliane ERJ, Susin LO, Folletto JL, Lermen NR, Wu VYJ, et al. Evolução do padrão de aleitamento materno. Rev Saúde Pública 2000; 34:143-8.

7. Passos MC, Lamounier JA, Silva CAM, Freitas SSN, Baudson MFR. Práticas da amamentação no Município de Ouro Preto, MG, Brasil. Rev Saúde Pública $2000 ; 34: 617-22$.

8. Vannuchi MTO, Monteiro CA, Réa MF, Andrade SM, Matsuo T. Iniciativa Hospital Amigo da Criança e aleitamento materno em unidade de neonatologia. Rev Saúde Pública 2004; 38:422-8.

9. Ramos CV, Almeida JAG, Alberto NSMC, Teles JBM, Saldiva SRDM. Diagnóstico da situação do aleitamento materno no Estado do Piauí, Brasil. Cad Saúde Pública 2008; 24:1753-62.

10. França GVA, Brunken GS, Silva SM, Escuder MM, Venâncio SI. Determinantes da amamentação no primeiro ano de vida em Cuiabá, Mato Grosso. Rev Saúde Pública 2007; 41:711-8.

11. Boccolini CS, Carvalho ML, Oliveira MIC, Leal MC, Carvalho MS. Fatores que interferem no tempo entre o nascimento e a primeira mamada. Cad Saúde Pública 2008; 24:2681-94.

12. Alves CRL, Goulart EMA, Colosimo EA, Goulart LMHF. Fatores de risco para o desmame entre usuárias de uma unidade básica de saúde de Belo Horizonte, Minas Gerais, Brasil, entre 1980 e 2004. Cad Saúde Pública 2008; 24:1355-67.

13. Mascarenhas MLW, Albernaz EP, Silva MB, Silveira RB. Prevalence of exclusive breastfeeding and its determiners in the first 3 months of life in the South of Brazil. J Pediatr (Rio J.) 2006; 82:289-94.

14. Baptista GH, Andrade AHHKG, Giolo SR. Fatores associados à duração do aleitamento materno em crianças de famílias de baixa renda da região sul da cidade de Curitiba, Paraná, Brasil. Cad Saúde Pública 2009; 25:596-604.
15. Schwartz K, D’Arcy HJ, Gillespie B, Bobo J, Longeway M, Foxman B. Factors associated with weaning in the first 3 months postpartum. J Fam Pract 2002; 51:439-44.

16. Dearden K, Altaye IM, Oliva MSJ, Morrow AL, Burkhalter BR. Determinants of optimal breast-feeding in peri-urban Guatemala City, Guatemala. Rev Panam Salud Pública 2002; 12:185-92.

17. Demidenko E. Sample size determination for logistic regression revisited. Stat Med 2007; 26:3385-97.

18. Demidenko E. Sample size and optimal design for logistic regression with binary interaction. Stat Med 2008; 27:36-46.

19. CLT - Consolidação das Leis do Trabalho. 25a Ed. São Paulo: Editora Saraiva; 1999.

20. World Health Organization. Indicators for assessing breastfeeding practices. Geneva: World Health Organization; 1991.

21. Ciaccia MCC, Ramos JLD, Issler H. Amamentação e trabalho da mulher: como conciliar? Rev Paul Pediatr 2003; 21:83-8.

22. Escobar AMU, Ogawa AR, Hiratsuka M, Kawashita MY, Teruya PY, Grisi S, et al. Aleitamento materno e condições socioeconômico-culturais: fatores que levam ao desmame precoce. Rev Bras Saúde Matern Infant 2002; 2:253-61.

23. Santiago LB, Bettiol H, Barbieri MA, Guttierrez MRP, Ciampo LAD. Incentivo ao aleitamento materno: a importância do pediatra com treinamento específico. J Pediatr (Rio J.) 2003; 79:504-12.

24. Barros FC, Victora CG. Maternal-child health in Pelotas, Rio Grande do Sul State, Brazil: major conclusions from comparisons of the 1982, 1993, and 2004 birth cohorts. Cad Saúde Pública 2008; 24 Suppl 3:S461-7.

25. Faleiros FTV, Trezza EMC, Carandina L. Aleitamento materno: fatores de influência na sua decisão e duração. Rev Nutr 2006; 19:623-30.

26. Silveira FJF, Lamounier JA. Fatores associados à duração do aleitamento materno em três municípios na região do Alto Jequitinhonha, Minas Gerais, Brasil. Cad Saúde Pública 2006; 22:69-77.

27. Beretta MIR, Frasson DA, Pacífico LHR, Denari FE. Avaliação do sistema de alojamento conjunto na maternidade D. Francisca Cintra Silva da Santa Casa de São Carlos-SP. Rev Latinoam Enferm 2000; 8:59-66.

28. Ichisato SMT, Shimo AKK. Revisitando o desmame precoce através de recortes da história. Rev Latinoam Enferm 2002; 10:578-85.

29. Venâncio SI, Escuder MML, Kitiko P, Rea MF, Monteiro CA. Freqüência e determinantes do aleitamento materno em municípios do Estado de São Paulo. Rev Saúde Pública 2002; 36:313-8.

30. Ransjö-Arvidson AB, Matthiesen AS, Lilja G, Nissen E, Widström AM, Uvnäs-Moberg K. Maternal analgesia during labor disturbs newborn behavior: effects on breastfeeding, temperature, and crying. Birth 2001; 28:5-12. 
31. Meirelles CAB, Oliveira MIC, Mello RR, Varela MAB, Fonseca VM. Justificativas para uso de suplemento em recém-nascidos de baixo risco de um Hospital Amigo da Criança. Cad Saúde Pública 2008; 24:2001-12.

32. Rea MF, Venâncio SI, Batista LE, Santos RG, Greiner T. Possibilidades e limitações da amamentação entre mulheres trabalhadoras formais. Rev Saúde Pública 1997; 31:149-56.

33. Damião JJ. Influência da escolaridade e do trabalho maternos no aleitamento materno exclusivo. Rev Bras Epidemiol 2008; 11:442-52.
34. Vianna RPT, Rea MF, Venâncio SI, Escuder MM. A prática de amamentar entre mulheres que exercem trabalho remunerado na Paraíba, Brasil: um estudo transversal. Cad Saúde Pública 2007; 23:2403-9.

35. Borges AL, Philippi ST. Opinião de mulheres de uma unidade de saúde da família sobre a quantidade de leite materno produzido. Rev Latinoam Enferm 2003; 11:287-92.

Recebido em 15/Mar/2010

Versão final reapresentada em 17/Jun/2010

Aprovado em 21/Jun/2010 